\title{
In vivo screening measurements with common radiation protection instruments
}

\author{
Siria Medici ${ }^{1,2,}$, Laurent Desorgher ${ }^{2}$, Pierre Carbonez ${ }^{1,3}$, Jérôme Damet $^{1,2,3}$, François \\ Bochud $^{2}$, and Andreas Pitzschke ${ }^{2}$ \\ ${ }^{1}$ European Organization for Nuclear Research (CERN), CH-1211 Geneva 23, Switzerland \\ ${ }^{2}$ Institute of Radiation Physics, Lausanne University Hospital Center, Rue du Grand-Pré 1, CH-1007 \\ Lausanne, Switzerland \\ ${ }^{3}$ Department of Radiology, University of Otago, 2 Riccarton Ave, Christchurch, New Zealand
}

Professionals working with unsealed radioactive sources and/or in the presence of contaminated or activated materials are at risk of incorporation of radionuclides. Two scenarios of internal contamination are possible: 1 ) in the framework of isolated operations, such as the maintenance of activated accelerator components and radioactive waste handling, or 2) during routine operations, such as isotope collections and manipulation of radiopharmaceuticals.

Our work aims to set the basis for a new monitoring program for internal contamination at CERN (where both exposure scenarios are present) by using conventional radiation protection instruments.

The monitoring program is inspired by the Swiss regulation and consists in screening measurements that aim to determine if a significant internal contamination occurred. The screening measurements are quick and simple and can usually be performed by the workers themselves. If a radionuclide-specific threshold is exceeded during a screening measurement, the worker must undergo a specific incorporation measurement to assess the intake activity that is then used for the evaluation of the committed dose. If the screening measurement is negative, no dose assessment is required. Therefore, the screening thresholds are set in order to guarantee that the committed effective dose does not exceed 1 $\mathrm{mSv} /$ year.

In our case, the screening measurements are performed as soon as possible after an isolated operation or periodically in the case of routine operations.

The development of the monitoring program includes the characterisation of conventional radiation protection instruments such as dose rate meters, contamination monitors and probes, to determine whether they are suitable to perform screening measurements. The characterisation is done using different isotopes to cover a wide photon energy range (124 $\mathrm{keV}-1.3 \mathrm{MeV}$ ). Instruments' characteristic limits are calculated accordingly to the ISO 11929 standard [1] and are provided for different background radiation levels. Simple and easily reproducible phantoms are used to link the measured quantity to the incorporated activity. The obtained experimental results are cross-validated with GEANT4 Monte Carlo simulations [2]. The minimum detectable activity is derived from the instrument's

*Corresponding author: siria.maria.chiara.medici@cern.ch 
characteristic limits. The biokinetic models recently published by the International Commission on Radiological Protection [3] are used to assess:

1) the minimum detectable committed effective dose according to the time elapsed between the manipulation and the measurement in the case of isolated operations,

2) the optimal compromise between screening threshold and screening interval duration in the case of routine operations.

Our results show that, for a given radionuclide inserted into the phantoms, the response of the different instruments varies up to one order of magnitude. Therefore, the instrument choice is crucial and has to be done according to the radionuclide that is going to be detected.

The suggested screening measurements allow to detect the presence of an internal contamination using conventional radiation protection instruments. For the radionuclides used during the characterisation, a screening measurement performed the day following an isolated operation allows the detection of an internal contamination leading to a committed effective dose in the order of tens up to a few hundreds of $\mu \mathrm{Sv}$, depending on the chosen instrument.

The methodology provides local radiation protection experts with the necessary tools to set a dedicated monitoring program for internal contamination.

In this presentation, we will explain the methodology used to implement in vivo screening measurements at CERN and show that it can be easily adapted to the needs of other research centres. We will present the performances of six commonly used radiation protection instruments and illustrate how to use them to perform screening measurements considering examples of real case exposure scenarios.

\section{References}

1. International Organization for Standardization, ISO Standard 11929 (2010).

2. J. Allison, et al, Nucl Instrum Methods Phys Res A, 835, 186-225 (2016).

3. International Commission on Radiological Protection, ICRP Publication 130, Ann. ICRP 44(2) (2015). 